\title{
Strain-level Screening of Human Gut Microbes Identifies Blautia Producta as a Novel Anti- hyperlipidemic Probiotic Via the Production of 12- methylmyristic Acid
}

\section{Chongming Wu ( $\nabla$ cmwu@implad.ac.cn )}

Chinese Academy of Medical Sciences and Peking Union Medical College https://orcid.org/0000-00032898-370X

\section{Wenyi Xu}

Beijing Quantihealth Technology Co., Ltd.,

Jiaqi Yu

Chinese Academy of Medical Sciences \& Peking Union Medical College Institute of Biomedical

Engineering

Zhuanyu Li

Beijing QuantiHealth Technology Co Ltd

\section{Yinghui Zhang}

Beijing QuantiHealth Technology Co Ltd

\section{Fang Zhang}

Chinese Academy of Medical Sciences \& Peking Union Medical College Institute of Medicinal Plant

Development

\section{Yanan Yang}

Chinese Academy of Medical Sciences \& Peking Union Medical College Institute of Medicinal Plant Development

\section{Qingshi Wang}

Beijing QuantiHealth Technology Co Ltd

\section{Yong Xie}

Chinese Academy of Medical Sciences \& Peking Union Medical College Institute of Medicinal Plant Development

\section{Bowen Zhao}

Beijing QuantiHealth Technology Co Ltd

\section{Research}

Keywords: Functional strain screening, Gut microbiota, Hyperlipidemia, Blautia producta, 12methylmyristic acid (12-MMA), G protein-coupled receptor 120 (GPR120) 
Posted Date: October 21st, 2021

DOI: https://doi.org/10.21203/rs.3.rs-989302/v1

License: (c) (i) This work is licensed under a Creative Commons Attribution 4.0 International License. Read Full License 


\section{Abstract}

Background: Compelling evidence has linked the commensal gut microbiota to human metabolic syndromes and provided new therapeutic potentials against diseases, such as hyperlipidemia. However, the precise regulatory effect of each bacterial species on human lipid homeostasis remains largely unknown.

Results: We set up a cell-based high-throughput screening platform and screened 2250 human gut bacterial strains from 186 species for the lipid-decreasing activity in HepG2 cells, in which 388 strains steadily inhibited lipid accumulation. Different strains in the same species usually displayed distinct lipidmodulatory actions, revealing an obvious strain-specificity. Blautia producta, Lactobacillus gasseri, and Bifidobacterium pseudolongum contained a much higher portion of hypolipidemic strains. Among all the tested strains, the mucosal bacterium Blautia producta exhibited the most potency to suppress lipid accumulation, and gavage of live Bl. producta effectively ameliorated hyperlipidemia in mice. 12Methylmyristic acid (12-MMA) was identified as an important active metabolite of BI. producta by pangenomics and comparative metabolomics, which exerted potent anti-hyperlipidemic effect in vivo and activated G protein-coupled receptor 120 (GPR120), thus stimulating white adipose tissue browning.

Conclusions: Together, these data reveal a previously unreported large-scale lipid-modulatory profile of gut microbes at the strain level, and raise the possibility of developing therapeutics based on Bl. producta and microbial metabolite 12-MMA to treat hyperlipidemia.

\section{Introduction}

Mounting progress in recognition of the inherent relationship between gut microbiota and host health not only reveals novel insights into the etiology of critical illnesses but also opens up new avenues for therapeutic interventions [1-5]. It is widely accepted that gut microbes play crucial roles in regulating host energy homeostasis and metabolism, especially lipid metabolism [6,7]. A comprehensive understanding of the lipid-modulatory landscape of various bacterial species will facilitate the development of novel therapeutics against lipid metabolic diseases. Multiple species, such as Lactobacillus spp,

Bifidobacterium spp, and Akkermansia muciniphila, are well-documented to possess beneficial effects on hyperlipidemia and non-alcoholic fatty liver disease (NAFLD) $[8,9]$. For gut microbiome researches, the cultured microbial repository can play indispensable role to facilitate the interpretation of microbial functions and host-microbiome interactions. Several large-scale cultivation efforts have been made to unravel the 'dark matters' of human gut microbiota [10-14]. However, our understanding of the human gut microbiome is still very insufficient. Which species or strains in our gut microbiota have therapeutic effects on lipid disorders remains poorly understood.

Free fatty acids (FFAs) are the best-characterized microbial metabolites to mediate various beneficial effects of gut microbiota on host metabolism and immunity $[3,15]$. Elevating short-chain fatty acids (SCFAs) production by gut microbes or direct supplementation of SCFAs in foods can significantly 
ameliorate dyslipidemia and NAFLD [16]. Besides, appropriate levels of saturated [17] or unsaturated long-chain fatty acids (LCFAs) [18] in the gut also contribute to maintain eubiosis, stabilize the gut barrier, and improve metabolic syndromes. Apart from straight-chain fatty acids, the branched-chain fatty acids, especially anteiso-fatty acids ( $a$ i-FAs), which contain an alkyl branch at the ante-penultimate carbon, are another main kind of microbial metabolites $[19,20]$. Compared with the straight-chain saturated counterparts, ai-FAs are featured with a marked decreased melting point [21], which may greatly increase membrane fluidity and change physiological functions like their straight-chain unsaturated counterparts. Although ai-FAs account for $10 \%$ of total lipids in soil, shea butter, camel milk, and mold [22], the study on physiological functions of ai-FAs is almost scarce.

Lipid metabolism is strictly regulated by multiple versatile regulators, for instance, $\mathrm{G}$ protein-coupled receptor 120 (GPR120) [23]. As a highly expressed protein in the gastrointestinal tract, lung, and adipose tissues, GPR120 performs various functions in a tissue-specific manner, such as regulating glucose metabolism and white fat browning [24]. Intestinal GPR120 activation stimulates the release of glucagonlike peptide-1 (GLP-1) and enhances glucose consumption [25]. Stimulating ciliary GPR120 in preadipocytes promotes adipocyte differentiation and drives the expansion of adipocyte numbers [26]. In GPR120-null mice, thermogenesis and fat browning are impaired [27]. The $\omega-3$ fatty acids such as

docosahexaenoic acid (DHA), a-linolenic acid (ALA), and eicosapentaenoic acid (EPA) are the most potent and common ligands of GPR120 [25,27]. Recently, Colosimo et al. found several saturated ai-FAs, such as 12-methyl tetradecanoic acid (also known as 12-methylmyristic acid) and 14-methyl hexadecanoic acid, can also potently activate GPR120, as the above mentioned polyunsaturated FAs do [28]. Therefore, aiFAs might be used as novel beneficial dietary nutrients to maintain host lipid/glucose homeostasis through activation of GPR120.

In the present study, we systemically screened the lipid-decreasing activities of 2250 human gut microbiota strains and, provided a large-scale lipid-modulatory profile at the strain level for the first time. Afterward, we corroborated the in vivo anti-hyperlipidemic effect of Blautia producta, a mucosal bacterium with the most potent suppressive efficacy in our screening, and identified12-methylmyristic acid (12-MMA) as an important active microbial metabolite to ameliorate hyperlipidemia. Furthermore, we demonstrated that 12-MMA could effectively activate GPR120 and consequently provoke GPR120downstream phenotypes, including improvement of glucose tolerance and up-regulation of white fat browning-associated marker genes. Thus, our findings advance the strain-level understanding of intestinal bacteria to regulate lipid metabolism and indicate that gut bacteria and their metabolites are a valuable source to develop novel medical agents for the prevention and treatment of hyperlipidemia.

\section{Materials And Methods}

\section{Generation of sterile spent culture broth filtrates}

All tested bacteria were from the human gut microbial library constructed by Quantihealth Co. Ltd, Beijing, China (https://microbe.quantibio.com/). More specifically, all strains were isolated from the mixture of 
fecal samples donated by healthy adults in Hainan province, China. Each strain was purely cultivated, identified by mass spectrometry, named with a unique code, and then cryopreserved at $-80^{\circ} \mathrm{C}$ refrigerator.

For screening assay, each bacterial clone was pin transferred from the cryopreserved tube onto a solid YCFA medium (Solarbio) plate and was grown for $24-48 \mathrm{~h}$ at $37^{\circ} \mathrm{C}$ under anaerobic conditions. A single colony was inoculated into liquid YCFA medium and cultured at $37^{\circ} \mathrm{C}$ for $48 \mathrm{~h}$ under anaerobic conditions to obtain the first generation (F1) bacterial solution. $10 \%(\mathrm{v} / \mathrm{v})$ of $\mathrm{F} 1$ bacterial solution was inoculated into fresh liquid YCFA medium and cultured at the same conditions for $48 \mathrm{~h}$ to generate F2 solution. Following the similar step as F2 production, the working bacterial solution F3 was further obtained from $10 \%(\mathrm{v} / \mathrm{v}) \mathrm{F} 2$. After centrifugation at $1,600 \times \mathrm{g}$ for $15 \mathrm{~min}$, the supernatant part was collected and then filtered using a Millipore filter $(0.22 \mu \mathrm{m})$. These sterile spent culture broth filtrates were used as screening samples in the study.

\section{Cellular lipid accumulation assay}

HepG2 cells were purchased from ATCC and were cultured in Dulbecco's modified Eagle's medium (DMEM, Thermo Fisher Scientific) supplemented with $10 \%$ fetal bovine serum (Thermo Fisher Scientific) at $37{ }^{\circ} \mathrm{C}$ with $5 \% \mathrm{CO}_{2}$. For lipid accumulation assay, HepG2 cells were seeded in 96 -well plates containing $100 \mu \mathrm{l}$ DMEM. When the confluence reached $85 \%$, the medium was replaced with $70 \mu \mathrm{l}$ fresh DMEM supplemented with $100 \mu \mathrm{M}$ oleic acid (OA, Sigma) and $30 \mu \mathrm{l}$ spent culture broth filtrate or YCFA medium. After incubation for 22-24 h, lipid accumulation was evaluated by oil red 0 staining or TG quantification kit (Solarbio) as described previously [57]. Each experiment ( $\mathrm{n}=8$ for oil red 0 staining, $\mathrm{n}=$ 4 for TG determination) was repeated in triplicate. Liquid YCFA medium and fenofibrate (10 $\mu \mathrm{M}$, Sigma) were used as the negative and positive control, respectively. Specifically, (1) for the large-scale bacteria screening, HepG2 cells were first stained with oil red $O$ solution at room temperature for $30 \mathrm{~min}$, and then DMSO was added to dissolve stains attached to cells, followed by measuring OD value at $358 \mathrm{~nm}$ in a microplate reader. Lipid-lowering rate of each examined bacterial strain was evaluated and ranked according to the calculation of [(YCFA OD 358 - Sample $\left.\left.\mathrm{OD}_{358}\right) / \mathrm{YCFA} \mathrm{OD}_{358}\right]^{\star} 100 \%$. (2) To confirm the lipid-lowering efficacy of Blautia producta, BODIPY staining and intracellular TG content were measured to corroborate the screening result according to the manufacturers' instructions.

\section{Animal experiment}

All the animal experiments were performed in accordance with the National Institutes of Health regulations for the care and use of animals in research. The protocol was approved by the medical ethics committee of Peking Union Medical College (Nos. YZS201904021; YZS201910013; YZS202105022). All efforts were made to minimize animal suffering.

(1) To assess the anti-hyperlipidemic effect of live Bl. producta, 24 male C57BL/6J mice (8-week-old, 20$24 \mathrm{~g}$, Vital River Laboratory Animal Technology) were divided into three groups with eight animals in each group. One group was used as blank control and continued to feed on normal chow (Chow group) while other groups were fed HFD (60\% kcal fat as indicated, Beijing HuaFuKang Bioscience). Animals on HFD 
were gavaged with BI. producta (HFD+Bl. producta group, $10^{9} \mathrm{CFU}$ per animal per day) or an equal volume of YCFA medium (HFD group). Bodyweight was assessed weekly. After 4 weeks of treatment, mice were fasted overnight, anesthetized in chambers saturated with isoflurane, then sacrificed by cardiac puncture. Stools for metagenomic analysis were collected on the day before animals were euthanized. Blood was drawn in $1.5 \mathrm{ml}$ centrifuge tubes, and sera were separated for estimation of serum levels of total triglyceride (TG), total cholesterol (TC), low-density lipoprotein cholesterol (LDL-C) and highdensity lipoprotein cholesterol (HDL-c) by respective kits (Nanjing jiancheng Bioengineering Institute). Liver samples were weighed and snap-frozen in liquid nitrogen for sequential biochemical analysis.

(2) To assess the dynamic impact of live Bl. producta on gut microbiota in mice, 16 male C57BL/6J mice (8-week-old, 20-24 g) were randomly divided into two groups, fed on HFD, and gavaged with Blautia producta (HFD+BI. producta group, $10^{9} \mathrm{CFU}$ per animal per day) or an equal volume of YCFA medium (HFD group) for 8 weeks. The fecal materials were obtained from each mouse on Days 1, 3, 7, 14 and 28 after treatment. At the end of the experiment, mice were fasted overnight, anesthetized with isoflurane, and sacrificed by cardiac puncture. Blood was drawn in $1.5 \mathrm{ml}$ centrifuge tubes, and sera were separated for parameters evaluation. Liver samples were weighed and snap-frozen in liquid nitrogen for sequential biochemical analysis or fixed in $4 \%$ paraformaldehyde (Solarbio) for histological analysis.

(3) To assess the anti-hyperlipidemic effect of 12-methylmyristic acid (12-MMA), 16 male C57BL/6J mice (8-week-old, 20-24 g) were randomly divided into two groups and fed HFD. The test group (HFD+12-MMA) was gavaged with 12-MMA (40 mg/kg, Sigma), while the HFD group was given an equal volume of solvent ( $1 \%$ tween $80+0.5 \%$ carboxymethyl cellulose sodium (CMC-Na, Sigma)) for four weeks. At the end of the experiment, mice were fasted overnight, anesthetized in chambers saturated with isoflurane and then sacrificed by cardiac puncture. Blood was drawn in $1.5 \mathrm{ml}$ centrifuge tubes, and sera were separated for parameters evaluation. Liver and fat samples were weighed and snap-frozen in liquid nitrogen for sequential biochemical analysis or fixed in $4 \%$ paraformaldehyde (Solarbio) for histological analysis.

\section{Histology and immunohistochemistry analysis}

The liver and fat tissues of each mouse were fixed in 4\% paraformaldehyde, embedded in paraffin, and cut into slides with a thickness of $4 \mu \mathrm{m}$. Liver and fat tissue sections were stained with hematoxylin and eosin (H\&E) for histological analysis. For oil red 0 staining, liver tissues from the same liver lobe were cut into small pieces, then the frozen sections were rinsed in distilled water and stained with $0.2 \%$ oil red 0 (Sigma) and 60\% 2-propanol (Sigma) for $10 \mathrm{~min}$ at $37^{\circ} \mathrm{C}$. For immunohistochemistry analysis, fat slides were rinsed in $0.01 \mathrm{~mol} / \mathrm{L}$ sodium citrate $(\mathrm{pH} \mathrm{6.0)}$ and heated for $20 \mathrm{~min}$ in a microwave to retrieve antigen. The sections were blocked in blocking buffer containing $5 \%$ goat serum, $2 \%$ BSA, $0.1 \%$ Triton X100 and $0.1 \%$ sodium azide in PBS, then incubated overnight with anti-UCP1 (Cell Signaling) by a dilution of $1: 100$ at $4^{\circ} \mathrm{C}$. After being washed twice in PBS, slices were incubated with secondary antibodies (Cell Signaling) for $1 \mathrm{~h}$ at room temperature. Slides were counterstained with H\&E. All digital pictures were acquired using an EVOS X1 microscopy (Thermo Fisher Scientific). 


\section{DNA extraction}

The microbial genomic DNA of fecal samples were extracted by DNeasy PowerSoil kit (QIAGEN) and subjected to $1 \%$ agarose gel electrophoresis for evaluation. Concentration and purity of microbial DNA were determined with NanoDrop 2000 UV-vis spectrophotometer (Thermo Fisher Scientific) and Qubit 3.0 fluorometer (Thermo Fisher Scientific).

\section{Shotgun sequencing}

The gut microbial composition was determined by shotgun sequencing of the fecal samples collected from each mouse. Libraries were prepared using KAPA HyperPlus Library Preparation kit (KAPA Biosystems) and quantified by KAPA Library Quantification Kits (KAPA Biosystems) following the manufacturer's instructions. Shotgun sequencing was performed on Illumina NovaSeq 6000 System (Illumina) at a depth of $1 \mathrm{~Gb}$. Cluster generation, template hybridization, isothermal amplification, linearization, blocking, denaturing and hybridization of the sequencing primers were performed according to the workflow indicated by Illumina.

As previously described [58], low-quality reads were removed from the raw data by using MOCAT2 Sequencing adapters were removed by using Cutadapt software (version v1.14,-m 30). Then SolexaQA package was used to remove the reads with a threshold of less than 20 or the length of less than 30bp. The reads which could be aligned with the mouse genome (Mus musculus, GRCm38) were cleaned by using SOAP aligner software (v2.21, -M $4-10$-v 10). The clean reads were assembled by SOAP de novo software (an iterative De-Bruijn Graph De Novo Assembler) using the parameters of -d 1,-M 3,-R ,-u,-F to get the scaftigs of at least 500bp. Genes were predicted using MetaGeneMark. A non-redundant gene catalogue was constructed with CD-HIT using the parameters of c 0.95 -aS 0.9. The clean reads were mapped onto the gene catalogue with the length of at least $100 \mathrm{bp}$ using BWA software to calculate the gene abundance.

\section{Sequencing data analysis}

a-Diversity was calculated by a vegan (2.5-6) package and presented by Shannon and Simpson indices. The principal coordinate analysis (PCOA) and nonmetric multidimensional scaling (NMDS) were calculated based on the Bray-Curtis distance using vegan (2.5-6) package. Microbial community composition was analyzed using Metaphlan2 software. Briefly, the query reads were mapped against the reference genomes in RefSeq database (version 82) with a 97\% identity threshold. The linear discriminant analysis (LDA) effect size (LEfSe) method (https://github.com/biobakery/lefse) was used to identify species that show statistically significant differential abundances among groups. Heat maps were generated by using the R package "heatmap".

\section{Pan-genomics}

Twelve reference genomes of $B I$. producta were used to perform pan-genomic analysis, in which seven genomes were downloaded from NCBI RefSe or GenBank database and five genomes were constructed 
by de novo sequencing of pure BI. producta cultures. Genes annotation was obtained using prokka software (https://github.com/tseemann/prokka). The pan-genomic analysis was performed by ppanggolin software (https://github.com/labgem/PPanGGOLiN) to acquire gene matrix, jaccard distance-based UPGMA clustering tree, and KEGG functional annotation.

\section{Metabolomics analysis}

The untargeted metabolomics profiling of the pure microbial culture was performed on the metabolomic platform of Shanghai Biotree biomedical technology Co. Ltd.

(1) Sample preparation: The frozen samples were kept on the dry ice-ethanol bath. About $50 \mathrm{mg}$ material was accurately weighed in an Eppendorf Safelock microcentrifuge tube, to which $25 \mathrm{mg}$ of pre-chilled zirconium oxide beads, $10 \mu \mathrm{l}$ of internal standard, and $50 \mu \mathrm{l}$ of $50 \%$ pre-chilled methanol were added for automated homogenization (BB24, Next Advance, Inc.). After centrifugation at $14,000 \mathrm{~g}$ and $4^{\circ} \mathrm{C}$ for 20 min (Microfuge 20R, Beckman Coulter, Inc., Indianapolis, IN, USA), the supernatant was carefully transferred to an autosampler vial (Agilent Technologies, Foster City, CA, USA). Each aliquot of $175 \mu \mathrm{l}$ of pre-chilled methanol/chloroform $(\mathrm{v} / \mathrm{v}=3 / 1)$ was added to the residue for the second extraction. After centrifugation at $14,000 \mathrm{~g}$ and $4^{\circ} \mathrm{C}$ for $20 \mathrm{~min}$, the supernatant was carefully transferred to an autosampler vial. All samples in autosampler vials were evaporated briefly to remove chloroform using a CentriVap vacuum concentrator (Labconco) and further lyophilized with a FreeZone freeze dryer equipped with a stopping tray dryer (Labconco).

(2) Chromatographic separation: Chromatographic separation was performed on the Agilent 1200 Series HPLC instrument (Agilent) combined with an ACE Excel 3 C18 column $(100 \mathrm{~mm} \times 2.1 \mathrm{~mm}, 3.0 \mu \mathrm{m})$, an autosampler, quaternary pump, and vacuum degasser. The separation conditions were as follows: flow rate of $350 \mu \mathrm{l} / \mathrm{min}$, column temperature of $35^{\circ} \mathrm{C}$, injection volume of $3 \mu \mathrm{l}$, sampler tray of $4{ }^{\circ} \mathrm{C}$, mobile phase $A$ (water $+0.1 \%$ formic acid), and mobile phase $B$ (acetonitrile). The gradient elution programs were performed as described below: 0-1.5 min, 2\% B; 1.5-4.0 min, 2-20\% B; 4.0-9.0 min, 20-60\% B; 9.0-18.0 min, 60-98\% B; 18.0-19.0 min, 98\% B; 19.0-19.5 min, $98-2 \% \mathrm{~B}$; and $19.5-21.5 \mathrm{~min}, 2 \% \mathrm{~B}$ in the positive ion mode; 0-1.5 min, 2\% B; 1.5-3.0 min, 2-25\% B; 3.0-7.0 min, 25-45\% B; 7.0-8.0 min, 45$60 \% \mathrm{~B} ; 8.0-15.0 \mathrm{~min}, 60-98 \% \mathrm{~B} ; 15.0-16.0 \mathrm{~min}, 98 \% \mathrm{~B} ; 16.0-17.5 \mathrm{~min}, 98-2 \% \mathrm{~B}$; and $17.5-19.0 \mathrm{~min}, 2 \%$ $B$ in the negative ion mode.

MS was performed on the Agilent 6530 Q-TOF mass spectrometer (Agilent) coupled with electrospray ionization (ESI) source with detection capability for both negative and positive ion modes. MassHunter Workstation software (Agilent) was employed for the system operation. The operation conditions of mass spectrometer were as follows: capillary temperature of $320^{\circ} \mathrm{C}$, capillary voltage of $4,000 \mathrm{~V}$ for positive ion mode and 3,500 V for negative ion mode; nebulizer of $35 \mathrm{psig}$; sheath gas flow rate of $12 \mathrm{~L} / \mathrm{min}$; collision energy of $35 \mathrm{eV}$; drying gas temperature of $300^{\circ} \mathrm{C}$ and drying gas flow rate of $6 \mathrm{~L} / \mathrm{min}$. The mass range was set from 50 to $1100 \mathrm{Da}$ with full scan mode. For the QC samples, pooled QC samples of data were acquired to supervise the real samples' variability in the overall analytic run. 


\section{Oral glucose tolerance test (OGTT)}

As previously described [57], before the OGTT test, mice were fasted for $6 \mathrm{~h}$ and then gavaged of $2 \mathrm{~g} / \mathrm{kg}$ glucose. The blood glucose concentration in the tail vein was monitored at 0, 30, 60, 90 and 120 min after glucose administration (p.o.) using a glucose meter (Roche).

\section{Quantitative real-time quantitative PCR (qRT-PCR) assay}

The inguinal fat tissue was used to evaluate the mRNA levels of key genes involved in fat browning. Total RNA extraction, first-chain cDNA synthesis, and quantitative PCR assays were performed as previously reported [59]. The primers used for each gene were listed in Additional file 2: Table S7.

\section{Fluorescence-based GPR120 activation assay}

To evaluate the activating effect of 12-MMA on GPR120, we set up a fluorescence-based GPR120 activation assay using STC-1 intestine endocrine cells (purchased from ATCC) stably transfected with a yellow fluorescent protein-based cAMP indicator Flamindo2 [60], as well as a detection test of GLP-1 secretion. (1) For GPR120 activation assay, cells were incubated with different concentrations of 12-MMA or myristic acid (MA, Sigma) or dimethyl sulfoxide (as a vehicle) for 20 min after transient transfection of GPR120-expressing plasmid. The fluorescence signal intensity (FL) were recorded by Tecan Infinite M1000Pro Microplate Reader at excitation $485 \mathrm{~nm}$ and emission $535 \mathrm{~nm}$. GPR120 activation was calculated as ( $\left.\mathrm{FL}_{12 \mathrm{MMA} \text { or } M A}-\mathrm{FL}_{\mathrm{DMSO}}\right) / \mathrm{FL}_{\mathrm{DMSO}}{ }^{*} 100 \%$. (2) The secreted level of glucagon-like peptide- 1 (GLP-1) in the culture medium was determined using mouse GLP-1 ELISA Kit (Solarbio) according to the manufacturer's instructions.

\section{Statistical analysis, graphing and figure assembly}

Data are presented as means \pm SEM. SPSS 17.0 and Prism 7 (Graphpad) were used for statistical analysis. The significance of group differences for normally distributed data was assessed by one-way ANOVA followed by Bonferroni post hoc tests. PERMANOVA (Permutational multivariate analysis of variance) was performed to evaluate the significance of group differences for PCoA and NMDS analysis. $P^{*}<0.05$ was considered statistically significant. All final figures were assembled using Adobe Illustrator.

\section{Results}

Gut bacteria exhibit diverse and strain-specific effects on intracellular lipid accumulation

To get a comprehensive insight into the roles of gut microbes on lipid metabolism, we set up a cell-based high-throughput screening platform to assess the lipid-modulatory activity of the conditioned culture medium of the individual gut bacterium at the strain level (Fig. 1a). A total of 2250 strains were screened for their modulation on OA-elicited intracellular lipid accumulation. These gut bacterial strains contained 5 phyla, 12 classes, 48 genera, and 186 species (Additional file 2: Table S1 and S2), covering most of 
the dominant genera in humans except Faecalibacterium and Akkermansia [29]. After three cycles of oilred 0 staining-based screening, 388 strains showed a steady lipid-lowering effect in which 294 strains were from Firmicutes, 76 from Actinobacteria, 13 from Proteobacteria, and 5 from Bacteroidetes (Fig. 1b; Additional file 2: Table S3). At the genus level, 8 genera provided more than 25 strains for screening in this test and their positive rates were ranked as Lactobacillus (24.48\%, 141/576), Clostridium $(19.44 \%$, 14/72), Lysinibacillus (17.83\%, 28/157), Bifidobacterium (16.63\%, 75/451), Bacillus (14.41\%, 84/583), Enterococcus $(9.85 \%, 13 / 132)$, Escherichia $(8.00 \%, 2 / 25)$, and Streptococcus $(5.88 \%, 2 / 34)$ (Fig. 1c). When focused on individual species with $\geq 25$ tested strains, Lactobacillus gasseri (43.88\%), Bifidobacterium pseudolongum (28\%) and Bifidobacterium catenulatum (25\%) showed higher positive rates, while Enterococcus faecails (8.82\%) and Escherichia coli (8\%) contained less lipid-lowering strains (Fig. 1d; Additional file 2: Table S4). These data provided several valuable reservoirs for seeking beneficial strains to maintain lipid homeostasis.

Interestingly, the positive rate rose sharply with screened strain numbers increasing. Although many species with $<5$ tested strains did not exhibit lipid-decreasing effect in these screenings, all species with $\geq 14$ tested strains provided at least one positive strain regardless of their taxonomy (Fig. 1e; Additional file 2: Table S2). Moreover, many species, such as B. adolescentis, L. gasseri, B. licheniformis contained lipid-increasing, lipid-lowering, and neutral strains at the same time (Table S3), suggesting an apparent strain-specific modulation on host lipid accumulation. Among all the tested bacterial species, Blautia producta is of the most notice because all five tested strains exhibited prominent lipid-decreasing activity with an efficacy comparable to fenofibrate, an effective lipid-lowering drug widely used in clinic ( Additional file 2: Table S2 and S3).

Bl. producta exerts potent anti-hyperlipidemic action

Blautia producta is a popular mucosal bacterium belonging to the Lachnospiraceae group [30]. Prior studies have demonstrated Bl. producta as a beneficial bacterial to resist pathogen invasion [31,32] and inhibit inflammation [33], but its anti-hyperlipidemic effect has not been investigated. We, therefore, systemically assessed the lipid-lowering effect and identified the active metabolite of $B I$. producta following the flow chart in Fig. 2a. The cell-based experiments showed that the conditioned culture medium of $B$ l. producta dose-dependently decreased the lipid accumulation in HepG2 cells, and its $30 \%(\mathrm{v} / \mathrm{v})$ conditioned culture medium displayed a lipid-lowering efficiency comparable to fenofibrate (Fig. 2b-e). To further confirm its hypolipidemic effect, we treated HFD-fed mice with live Bl. producta via continuous gavage of $10^{9} \mathrm{CFU}$ per animal per day for 4 weeks. Live Bl. producta significantly suppressed HFD-induced body weight gain (Fig. 2f-g) and fat deposition (Fig. 2h), and alleviated hyperlipidemia (Fig. 2i), thus leading to the improvement of liver steatosis (Fig. 2j-k). When prolonged the treatment period to 8 weeks, gavage of live Bl. producta displayed a similar pharmacological efficiency on the aforementioned indicators, including body weight, hyperlipidemia and liver steatosis (Additional file 1: Fig.S1). These results collectively demonstrated the prominent lipid-lowering effects of Bl. producta. 
We further inspected the impact of live BI. producta on host gut microbiota. Oral administration of Bl. producta did not change the diversity of the gut microbes but shifted its compositional structure (Fig. 3ad). At the genus level, the most striking change was that the dramatic decrease of Akkermansia resulted from HFD feeding was restored by $B$ l. producta (Fig. 3e). More specifically, the relative abundance of Akkermansia muciniphila was significantly increased by Bl. producta (Fig. 3f). At the same time, beneficial mucosal bacteria such as Bifidobacterium animalis, Lactobacillus pentosus and Lactobacillus sakei, were elevated while opportunistic pathogens Desulfovibrio piger, Desulfovibrio sp. G11 were declined in response to $B$ l. producta administration (Fig.3f). Lefse analysis also revealed that Erysipelotrichales and Verrucomicrobiales were enriched after gavage of Bl. producta (Fig. 3g,h).

However, gavage of live Bl. producta did not increase its relative abundance in feces (Fig. 3f). To confirm this observation, we performed an extra dynamic detection by repeating the animal experiment of $B I$. producta and collecting feces on Day $1,3,7,14$ and 28 . The results displayed that oral administration of Bl. producta dynamically shifted the overall structure of gut microbiota (Additional file 1: Fig.S2a). BI. producta showed the greatest impaction on gut microbiome at Day 1 , then the influence turned weaker but it still reached significance on the $28^{\text {th }}$ day after treatment (Additional file 1: Fig.S2a). The in vivo abundance of Bl. producta was also regulated in a dynamic manner. Gavage of exogenous BI. producta significantly increased the fecal content of BI. producta on Days 1 and 7 , while this stimulation gradually disappeared afterward and the abundance of fecal BI. producta on Day 28 in BI. producta-treated mice was mildly less than that in control mice (Additional file 1: Fig.S2b), which was similar to our prior data. To certify the uniform of these two experiments, we quantified the abundance of several key genera and species in the fecal samples collected on the $28^{\text {th }}$ day after treatment and compared them with previous results. Gavage of Bl. producta showed the same modulatory trend on Akkermansia spp., B. animalis, L. pentosus, L. sakei, D. piger and Desulfovibrio sp. G11 in both experiments (Fig.2e,f; Additional file 1: Fig.S2c,d). These findings indicated that $B I$. producta is a beneficial microorganism with poor colonizing capacity. Hence, we guessed $B I$. producta might exert lipid-lowering action via secretion of active compounds rather than stimulating intestinal Bl. producta growth.

12-Methylmyristic acid (12-MMA) is an important active metabolite of $B$ l. producta

To identify the active metabolites that mediate the beneficial effect of Bl. producta, pan-genomic analysis and UPLC-MS/MS-based untargeted metabolomics were conducted. The pan-genomic analysis included 5 new genomes of $B$. producta by de novo sequencing, namely I2DA, ID8A, ID9B, I24C and I31A, and 7 reference genomes from public databases. The rank of lipid-lowering efficiency of $5 \mathrm{Bl}$. producta strains was I2DA>ID8A>|D9B $>|24 C>| 31 A$. The 5 lipid-lowering strains clustered in two neighbor branches (Additional file 1: Fig.S3a) and genes involved in lipid metabolism were uniformly enriched in these strains (Additional file 1: Fig.S3b), implying that active lipid metabolism might be a unique feature the effective Bl. producta strains.

We further performed untargeted metabolomics by using UPLC-MS/MS method. To find the preferentially produced metabolites by $B$. producta, 500 typical strains were selected from 2250 bacteria tested in our 
study, which covered all the genera screened in this study. The 500 strains were cultured under the same condition for lipid-lowering activity assay, and their monocultures were pooled with equal weight as an entirety mixture. The signal strength of each recognized metabolite in Bl. producta was compared with that in the entirety mixture, to assess the priority of $B l$. producta in producing each metabolite. A total of 614 metabolites were identified and 13 metabolites were highly ( $>2.8$ fold) generated by $B I$. producta over the entirety (Fig. 4a; Additional file 2: Table S5). 12-Methylmyristic acid (12-MMA) was the top priority product with a yield of 26.4 times over the whole. Therefore, 12-MMA was the most preferentially produced metabolite by $B I$. product, although it is unnecessary to be the most abundant metabolite in this strain. Generally, an effective approach to identify the active metabolite of a particular species is to find different strains with distinct pharmacological efficiencies and correlate the abundance of their metabolite with the differential effects. Because all tested BI. producta strains were highly effective, it is impossible to identify key active metabolites following this strategy. We, therefore, modified the method by selecting another gut bacterial species harboring both lipid-decreasing and increasing strains as a reference. In our screening, Bacilli licheniformis virtually met the aforementioned requirements and the great majority of strains exhibited metabolite profiles highly resembling Bl. producta. As revealed by PLSDA and Hierarchical Clustering, the effective (lipid-decreasing) and ineffective (lipid-increasing) strains were markedly separated (Fig. 4b-c). Volcano plot showed that several metabolites differently distributed among bacteria, in which 12-MMA and anandamide were enriched in effective strains, whilst pyrrolidonecarboxylic acid was higher in ineffective ones (Fig. 54). Furthermore, random forest analysis clearly exhibited that 12-MMA was the most critical metabolite to distinguish the effective bacteria from the ineffective ones (Fig. 4e; Additional file 2: Table S6).

\section{2-MMA effectively alleviates hyperlipidemia}

In light of 12-MMA as a critical metabolite candidate of Bl. producta, we treated HFD-fed mice with 12MMA for four weeks to verify its anti-hyperlipidemic effect. Oral administration of 12-MMA significantly inhibited HFD-induced body weight gain and fat deposit (Fig. 5a-b). High serum lipids levels were remarkably reduced by 12-MMA (Fig. 5c). Consequently, liver steatosis was also alleviated after 12-MMA treatment, as revealed by lipid quantification and oil-red 0 staining (Fig. $5 \mathrm{~d}-\mathrm{e})$. These results demonstrated that 12-MMA is an active metabolite of Bl. producta to alleviate hyperlipidemia.

Besides the beneficial effect on hyperlipidemia, supplementation of 12-MMA also improved oral glucose tolerance (Fig. 5f), indicating the beneficial roles in both lipid and glucose homeostasis. The 12-MMAtreated mice displayed multiple multilocular adipocytes in inguinal white fat (iWAT), representing a typical characteristic of WAT browning (Fig. $5 \mathrm{~g}$ ). Consistently, the expression of uncoupling protein 1 (Ucp1) and the transcription levels of crucial thermogenic genes in the iWAT were significantly upregulated (Fig. 5h-i), indicating a stimulatory effect of 12-MMA on WAT browning. Moreover, oral administration of 12-MMA significantly increased the serum level of glucagon-like peptide-1 (GLP-1), a popular downstream peptide of GPR120 (Fig. 5j). Recent reports showed that GPR120 activation contributes to both glucose consumption and WAT browning [27]. We, therefore, evaluated the influence of 12-MMA on GPR120 activity by a fluorescence-based assay [45] in STC-1 intestine endocrine cells. 
Both 12-MMA and its counterpart myristic acid (MA) drastically strengthened GPR120 activity in a dosedependent manner (Fig. 5k), the secretion of GLP-1 was enhanced as well (Fig. 5l). These findings provided a potential mechanism by which 12-MMA exerts therapeutic efficacy against hyperlipidemia.

\section{Discussion}

Over the past decade, it has been generally accepted that the gut microbiota plays vital role in regulating host lipid metabolism [34]. However, due to the lack of species/strain-level investigations, the exact effect of individual species on lipid homeostasis remains poorly understood. Here we, for the first time, displayed a considerable functional profile regarding lipid-modulatory capacities of human gut microbiota and identified a key active microbial metabolite, 12-MMA, which triggers GPR120 activation and thereby ameliorates hyperlipidemia and obesity.

Currently, intensive studies have illustrated the beneficial effects of the gut microbiome, providing an essential rationale to develop novel therapeutic approaches based on the vast bacteria reservoir. Several groups have performed screening investigations on gut microbes to discover more functional connections between the gut microbiome and hosts, such as carbohydrate degradation [34] and antibiotic resistance [35]. In the present study, we set up a high-throughput screening platform, aiming to seek the functional human gut bacteria with lipid-decreasing effects. A total of 2250 strains were screened, covering 186 species and representing an essential part of human gut microbiota. These screenings not only first display a large-scale functional profile of intestinal bacteria at the strain level, but also provide a systematical understanding of hypolipidemic strains and several bacteria pools with massive potentials for therapeutic exploitation. According to our analysis, 388 out of the 2250 strains (about 17\%) steadily suppressed the lipid accumulation in three rounds of evaluations. Firmicutes (17.81\%) and Actinobacteria (16.59\%) possess a greater portion of lipid-decreasing bacteria than Bacteroidetes (13.16\%) and Proteobacteria (12.87\%). At the genus level, Blautia (100\%), Providencia (33.33\%), Pseudomonas (28.57\%), Lactobacillus (24.48\%), Bacteroides (22.22\%), Mitsuokella (20.00\%), Clostridium (19.44\%), Lysinibacillus (17.83\%), Bifidobacterium (16.63\%) and Bacillus (14.41\%) were the top 10 genera containing lipid-lowering strains. Among them, Lactobacillus and Bifidobacterium are two well-documented genera comprising multiple species with well-proven beneficial effects on hyperlipidemia and NAFLD [36-39]. In our study, more than $60.99 \%$ of positive Lactobacillus strains were from $L$. gasseri, a well-known species with great potential to help with weight loss in humans and rodents $[40,41]$. Another notable species is Lysinibacillus sphaericus, $16.83 \%(17 / 101)$ of its tested strains stably inhibited lipid accumulation though the biological activity of this species is rarely reported. Hence, $L$. gasseri and $L$. sphaericus could be valuable sources to deeply mining for hypolipidemic strains. Moreover, we conclude from the screening analysis that strains belonging to the same species usually function diversely, obviously presenting strain-specific regulatory roles on host lipid metabolism. Therefore, close attention should be paid to this when future researches are arranged to explore active functional bacteria or probiotics. 
One important finding of this work is the identification of Bl. producta, which is a mucosal bacterium belonging to the Lachnospiraceae group [42]. In our screening, BI. producta is of the most attraction because all tested strains robustly decreased lipid accumulation with comparable efficacy to the positive drug fenofibrate. Analogously, gavage of live Bl. producta isolated from human feces was effective to suppress HFD-induced body weight gain, decrease serum levels of lipids, and ameliorate liver steatosis in mice, exhibiting potent efficacy against hyperlipidemia. Prior studies have revealed that Bl. producta plays an essential role in the colonization resistance to the invasion of Vancomycin Resistant Enterococci (VRE) $[43,44]$ and exhibits anti-inflammatory effect in HT-29 intestinal epithelial cells [45]. Besides, recent studies revealed that Blautia spp. are closely associated with the improvement in glucose and lipid homeostasis by drugs, such as metformin [46], resveratrol [47] and erythrocyte n-6 polyunsaturated fatty acids [48], yet the specific species are still not determined. Our work not only demonstrates the antihyperlipidemic effect of $B l$. producta in the mouse model, paving a path for the development of $B$. producta as a novel probiotics, but also releases an inspiring clue that $B I$. producta might be a candidate to mediate the lipid-modulatory effects of some drugs.

Another interesting finding of this study is that we identified 12-MMA as a key active metabolite of $B /$. producta and verified its marked efficacy against hyperlipidemia in mice. Apart from SCFAs, increasing evidence has documented the contributing roles of LCFAs in maintaining the balance of energy metabolism $[49,50], 12-M M A$ found in our work again highlights the importance of LCFAs, together with another LCFA, myristoleic acid (MA), which reduces obesity through brown fat activation [51]. Moreover, 12-MMA belongs to anteiso-fatty acids (ai-FAs), a type of fatty acid that contain an alkyl branch at the ante-penultimate carbon. At present, few literatures are released regarding the physiological functions of ai-FAs. Colosimo et al. revealed that several ai-FAs could activate GPCRs as the polyunsaturated FAs do [28], suggesting the possibility that ai-FAs may act like the unsaturated FAs. Previous studies have shown that some ai-FAs can be major constituents in bacteria and 12-MMA, also known as 12-

methyltetradecanoic acid, accounting for $25-30 \%$ of the total fatty acids in Bacillus megaterium [52, 53]. Thus, 12-MMA might be indispensable for the growth of some species, and it could be constantly produced by the bacterial both in vitro or in vivo, although this needs further certification. This provides an explanation for the observation that 12-MMA was identified from the pure culture, but it was as effective as the whole bacterial strain in the animal experiment. Overall, the identification of 12-MMA as an active metabolite to improve hyperlipidemia widens our knowledge of ai-FAs. However, we currently cannot exclude the potential that other metabolites of Bl. producta also possess potent antihyperlipidemic effect, which is worth further investigation in the future.

Besides hyperlipidemia amelioration, 12-MMA also dose-dependently stimulated GPR120 and improved oral glucose tolerance. It has been well-acknowledged that GPR120 activation can effectively provoke brown fat activation and WAT browning $[27,54]$. Correspondingly, oral administration of 12-MMA remarkably up-regulated key thermogenic factors and elicited many multilocular adipocytes in iWAT, a typical phenotype of WAT browning. In light of the similar characteristics of anteiso- and unsaturated-FAs counterparts (12-MMA/MA), we speculate that MA may activate GPR120 as well, consequently resulting in BAT activation and improved obesity [51]. Of note, G-protein coupled receptors (GPCRs) are, to date 
quite successful therapeutic targets for various diseases [55, 56], so 12-MMA might be a potential candidate against hyperlipidemia by targeting GPR120, yet massive further investigation is awaited to verify this conjecture.

\section{Conclusions}

In the present study, we have first displayed a large-scale lipid-modulatory profile of gut microbes at the species/strain level and demonstrated BI. producta as a novel probiotic to exert prominent antihyperlipidemic effect via 12-MMA-GPR120-WAT browning axis to improve hyperlipidemia and obesity. This work opens up new avenues to systematically understand the modulatory roles of gut microbiota and provides possible therapeutic exploitation based on the gut microbiome and derived metabolites.

\section{Declarations}

\section{Authors' contributions}

CW and BZ conceived the project, designed the experiments. WX and JY performed most of the experiments with the help of $F Z, Y Y, Z L, Y Z, Y X$ and $Q W$. CW and WX prepared figures and wrote the manuscript. BZ and ZL provided the gut bacteria samples. All authors discussed the manuscript, commented on the project and contributed to manuscript preparation.

\section{Funding}

This work was supported financially by the National Natural Science Foundation of China (81673663 and 81973217) to CMW, the Non-profit Central Research Institute Fund of Chinese Academy of Medical Sciences (2018RC350014), CAMS Innovation Fund for Medical Sciences (CIFMS) (2016-I2M-3-015) to CW.

\section{Availability of data and materials}

The sequencing raw data and genome sequences in the present study have been deposited in the National Center for Biotechnology Information (NCBI) database, and the project numbers are PRJNA771420, PRJNA771488 and PRJNA771491. Other data relevant to the study are included in the article or uploaded as supplementary files.

\section{Ethics approval and consent to participate}

All animal experiments were performed in accordance with the National Institutes of Health regulations for the care and use of laboratory animals. The protocols used in the study were approved by the medical ethics committee of Peking Union Medical College (Nos. YZS201904021; YZS201910013; YZS202105022).

\section{Consent for publication}


Not applicable.

\section{Competing interests}

WX, ZL,YZ, QW and BZ are employees of Beijing QuantiHealth Technology Co., Ltd. The other authors declare they have no competing interests.

\section{Author details}

${ }^{1}$ Pharmacology and Toxicology Research Center, Institute of Medicinal Plant Development, Chinese Academy of Medical Sciences \& Peking Union Medical College, Beijing, 100193, China.

${ }^{2}$ Beijing QuantiHealth Technology Co., Ltd., Beijing, 100070, China.

\section{References}

1. Cryan JF, O'Riordan KJ, Sandhu K, Peterson V, Dinan TG. The gut microbiome in neurological disorders. The Lancet. Neurology 19, 179-194.

2. O'Toole PW, Jeffery IB. Gut microbiota and aging. Science (New York, N.Y.) 350, 1214-1215.

3. Rooks MG, Garrett WS. Gut microbiota, metabolites and host immunity. Nature reviews. Immunology $16,341-352$.

4. Roy S, Trinchieri G. Microbiota: a key orchestrator of cancer therapy. Nature reviews. Cancer17, 271285.

5. Valdes AM, Walter J, Segal E, Spector TD. Role of the gut microbiota in nutrition and health. BMJ (Clinical research ed.) 361, k2179.

6. Canfora EE, Meex RCR, Venema K, Blaak EE. Gut microbial metabolites in obesity, NAFLD and T2DM. Nature reviews. Endocrinology 15, 261-273.

7. Leung C, Rivera L, Furness JB, Angus PW. The role of the gut microbiota in NAFLD. Nature reviews. Gastroenterology \& hepatology 13, 412-425.

8. Cani PD. Microbiota and metabolites in metabolic diseases. Nature reviews. Endocrinology 15, 6970.

9. Canfora EE, Meex RCR, Venema K, Blaak EE. Gut microbial metabolites in obesity, NAFLD and T2DM. Nature reviews. Endocrinology 15, 261-273.

10. Liu C, Du M-X, Abuduaini R, Yu H-Y, Li D-H, Wang Y-J, et al. Enlightening the taxonomy darkness of human gut microbiomes with a cultured biobank. Microbiome 9, 1-29.

11. Zou Y, Xue W, Luo G, Deng Z, Qin P, Guo R, et al. 1,520 reference genomes from cultivated human gut bacteria enable functional microbiome analyses. Nat. Biotechnol. 37, 179-185.

12. Poyet M, Groussin M, Gibbons S, Avila-Pacheco J, Jiang X, Kearney S, et al. A library of human gut bacterial isolates paired with longitudinal multiomics data enables mechanistic microbiome research. Nat. Med. 25, 1442-1452. 
13. Browne HP, Forster SC, Anonye BO, Kumar N, Neville BA, Stares MD, et al. Culturing of 'unculturable'human microbiota reveals novel taxa and extensive sporulation. Nature 533, 543-546.

14. Forster SC, Kumar N, Anonye BO, Almeida A, Viciani E, Stares MD, et al. A human gut bacterial genome and culture collection for improved metagenomic analyses. Nat. Biotechnol. 37, 186-192.

15. Koh A, De Vadder F, Kovatcheva-Datchary P, Bäckhed F. From Dietary Fiber to Host Physiology: ShortChain Fatty Acids as Key Bacterial Metabolites. Cell 165, 1332-1345.

16. Canfora EE, Jocken JW, Blaak EE. Short-chain fatty acids in control of body weight and insulin sensitivity. Nature reviews. Endocrinology 11, 577-591.

17. Chen P, Torralba M, Tan J, Embree M, Zengler K, Starkel P, et al. Supplementation of saturated longchain fatty acids maintains intestinal eubiosis and reduces ethanol-induced liver injury in mice. Gastroenterology 148, 203-214 e216.

18. Quan LH, Zhang C, Dong M, Jiang J, Xu H, Yan C, et al. Myristoleic acid produced by enterococci reduces obesity through brown adipose tissue activation. Gut.

19. Eibler D, Seyfried C, Kaffarnik S. \& Vetter W. anteiso-Fatty Acids in Brussels Sprouts (Brassica oleracea var. gemmifera L.): Quantities, Enantioselectivities, and Stable Carbon Isotope Ratios. J. Agric. Food Chem. 63, 8921-8929.

20. Eibler D, Seyfried C, Vetter W. Enantioselectivity of anteiso-fatty acids in hitherto uninspected sample matrices. Journal of chromatography. B, Analytical technologies in the biomedical and life sciences 1061-1062, 233-239.

21. Kaneda T. Iso- and anteiso-fatty acids in bacteria: biosynthesis, function, and taxonomic significance. Microbiol Rev 55, 288-302.

22. Eibler D, Seyfried C, Vetter W. Enantioselectivity of anteiso-fatty acids in hitherto uninspected sample matrices. Journal of chromatography. B, Analytical technologies in the biomedical and life sciences 1061-1062, 233-239.

23. Kimura I, Ichimura A, Ohue-Kitano R, Igarashi M. Free Fatty Acid Receptors in Health and Disease. Physiol. Rev. 100, 171-210.

24. Im D-S. FFA4 (GPR120) as a fatty acid sensor involved in appetite control, insulin sensitivity and inflammation regulation. Mol. Aspects Med. 64.

25. Hirasawa A, Tsumaya $K$, Awaji T, Katsuma S, Adachi T, Yamada M, et al. Free fatty acids regulate gut incretin glucagon-like peptide-1 secretion through GPR120. Nature medicine 11, 90-94.

26. Hilgendorf KI, Johnson CT, Mezger A, Rice SL, Norris AM, Demeter J, et al. Omega-3 Fatty Acids Activate Ciliary FFAR4 to Control Adipogenesis. Cell 179, 1289-1305 e1221.

27. Quesada-Lopez T, Cereijo R, Turatsinze JV, Planavila A, Cairo M, Gavalda-Navarro A, et al. The lipid sensor GPR120 promotes brown fat activation and FGF21 release from adipocytes. Nature communications 7, 13479.

28. Colosimo DA, Kohn JA, Luo PM, Piscotta FJ, Han SM, Pickard AJ, et al. Mapping Interactions of Microbial Metabolites with Human G-Protein-Coupled Receptors. Cell host \& microbe 26, 273-282 
e277.

29. Arumugam M, Raes J, Pelletier E, Le Paslier D, Yamada T, Mende DR, et al. Enterotypes of the human gut microbiome. Nature 473, 174-180.

30. Maru BT, Munasinghe PC, Gilary H, Jones SW, Tracy BP. Fixation of CO2 and CO on a diverse range of carbohydrates using anaerobic, non-photosynthetic mixotrophy. FEMS Microbiol. Lett. 365, fny039.

31. Caballero S, Kim S, Carter RA, Leiner IM, Sušac B, Miller L, et al. Cooperating commensals restore colonization resistance to vancomycin-resistant Enterococcus faecium. Cell host \& microbe 21, 592602. e594.

32. Kim SG, Becattini S, Moody TU, Shliaha PV, Littmann ER, Seok R, et al. Microbiota-derived lantibiotic restores resistance against vancomycin-resistant Enterococcus. Nature 572, 665-669.

33. Pathmanathan SG, Lawley B, McConnell M, Baird MA, Tannock GW. Gut bacteria characteristic of the infant microbiota down-regulate inflammatory transcriptional responses in HT-29 cells. Anaerobe 61, 102112.

34. Zeevi D, Korem T, Godneva A, Bar N, Kurilshikov A, Lotan-Pompan M, et al. Structural variation in the gut microbiome associates with host health. Nature 568, 43-48.

35. Versluis D, de JBGT, Zoetendal, Passel EG M \& Smidt H. High throughput cultivation-based screening on porous aluminum oxide chips allows targeted isolation of antibiotic resistant human gut bacteria. Plos one 14, e0210970.

36. Naudin CR, Maner-Smith K, Owens JA, Wynn GM, Robinson BS, Matthews JD, et al. Lactococcus lactis Subspecies cremoris Elicits Protection Against Metabolic Changes Induced by a Western-Style Diet. Gastroenterology 159.

37. Zhang X, Coker OO, Chu ES, Fu K, Lau HCH, Wang Y-X, et al. Dietary cholesterol drives fatty liverassociated liver cancer by modulating gut microbiota and metabolites. Gut.

38. Scorletti E, Afolabi PR, Miles EA, Smith DE, Almehmadi A, Alshathry A, et al. Synbiotics Alter Fecal Microbiomes, But Not Liver Fat or Fibrosis, in a Randomized Trial of Patients With Nonalcoholic Fatty Liver Disease. Gastroenterology 158.

39. Koopman N, Molinaro A, Nieuwdorp M, Holleboom AG. Review article: can bugs be drugs? The potential of probiotics and prebiotics as treatment for non-alcoholic fatty liver disease. Aliment. Pharmacol. Ther. 50, 628-639.

40. Bauer PV, Duca FA, Waise TMZ, Dranse HJ, Rasmussen BA, Puri A, et al. Lactobacillus gasseri in the Upper Small Intestine Impacts an ACSL3-Dependent Fatty Acid-Sensing Pathway Regulating WholeBody Glucose Homeostasis. Cell Metab 27, 572-587 e576.

41. Kim J, Yun JM, Kim MK, Kwon O, Cho B. Lactobacillus gasseri BNR17 supplementation reduces the visceral fat accumulation and waist circumference in obese adults: a randomized, double-blind, placebo-controlled trial. J. Med. Food 21, 454-461.

42. Maru BT, Munasinghe PC, Gilary H, Jones SW, Tracy BP. Fixation of CO2 and CO on a diverse range of carbohydrates using anaerobic, non-photosynthetic mixotrophy. FEMS microbiology letters 365. 
43. Caballero S, Kim S, Carter RA, Leiner IM, Susac B, Miller L, et al. Cooperating Commensals Restore Colonization Resistance to Vancomycin-Resistant Enterococcus faecium. Cell host \& microbe 21, 592-602 e594.

44. Kim SG, Becattini S, Moody TU, Shliaha PV, Littmann ER, Seok R, et al. Microbiota-derived lantibiotic restores resistance against vancomycin-resistant Enterococcus. Nature 572, 665-669.

45. Pathmanathan SG, Lawley B, McConnell M, Baird MA, Tannock GW. Gut bacteria characteristic of the infant microbiota down-regulate inflammatory transcriptional responses in HT-29cells. Anaerobe 61, 102112.

46. Tong X, Xu J, Lian F, Yu X, Zhao Y, Xu L, et al. Structural Alteration of Gut Microbiota during the Amelioration of Human Type 2 Diabetes with Hyperlipidemia by Metformin and a Traditional Chinese Herbal Formula: a Multicenter, Randomized, Open Label Clinical Trial. mBio 9.

47. Wang P, Gao J, Ke W, Wang J, Li D, Liu R, et al. Resveratrol reduces obesity in high-fat diet-fed mice via modulating the composition and metabolic function of the gut microbiota. Free radical biology \& medicine 156, 83-98.

48. Miao Z, Lin JS, Mao Y, Chen GD, Zeng FF, Dong HL, et al. Erythrocyte n-6 Polyunsaturated Fatty Acids, Gut Microbiota, and Incident Type 2 Diabetes: A Prospective Cohort Study. Diabetes care.

49. Fedorenko A, Lishko PV, Kirichok Y. Mechanism of fatty-acid-dependent UCP1 uncoupling in brown fat mitochondria. Cell 151, 400-413.

50. Nakamura MT, Yudell BE, Loor JJ. Regulation of energy metabolism by long-chain fatty acids. Prog. Lipid Res. 53, 124-144.

51. Quan L-H, Zhang C, Dong M, Jiang J, Xu H, Yan C, et al. Myristoleic acid produced by enterococci reduces obesity through brown adipose tissue activation. Gut 69, 1239-1247.

52. Kaneda T. Iso-and anteiso-fatty acids in bacteria: biosynthesis, function, and taxonomic significance. Microbiol. Rev. 55, 288-302.

53. Yudkin M. Isolation and analysis of the protoplast membrane of Bacillus megaterium. Biochem. J. 98, 923.

54. Paschoal VA, Walenta E, Talukdar S, Pessentheiner AR, Osborn O, Hah N, et al. Positive Reinforcing Mechanisms between GPR120 and PPARgamma Modulate Insulin Sensitivity. Cell metabolism 31, 1173-1188 e1175.

55. Davenport AP, Scully CCG, de Graaf C, Brown AJH, Maguire JJ. Advances in therapeutic peptides targeting G protein-coupled receptors. Nature reviews. Drug discovery 19, 389-413.

56. Congreve M, de Graaf C, Swain NA, Tate CG. Impact of GPCR Structures on Drug Discovery. Cell 181, 81-91.

57. Qi G, Zhou Y, Zhang X, Yu J, Li X, Cao X, et al. Cordycepin promotes browning of white adipose tissue through an AMP-activated protein kinase (AMPK)-dependent pathway. Acta Pharm Sin B 9, 135-143.

58. Xu W, Chen T, Pei Y, Guo H, Li Z, Yang Y, et al. Characterization of Shallow Whole-Metagenome Shotgun Sequencing as a High-Accuracy and Low-Cost Method by Complicated Mock Microbiomes. 
Frontiers in Microbiology 12.

59. Wu C, Feng J, Wang R, Liu H, Yang H, Rodriguez PL, et al. HRS1 acts as a negative regulator of abscisic acid signaling to promote timely germination of Arabidopsis seeds. PLOS One 7, e35764.

60. Odaka H, Arai S, Inoue T, Kitaguchi T. Genetically-encoded yellow fluorescent cAMP indicator with an expanded dynamic range for dual-color imaging. PLoS One 9, e100252.

\section{Figures}


a

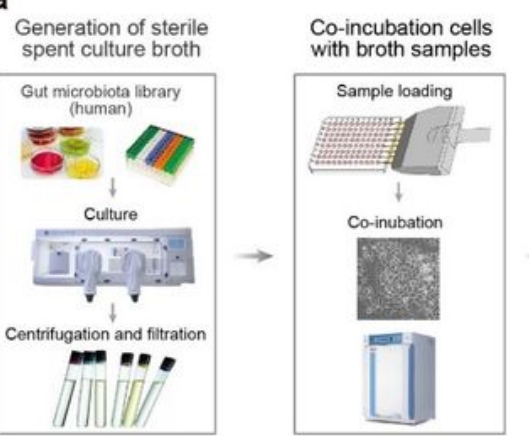

C

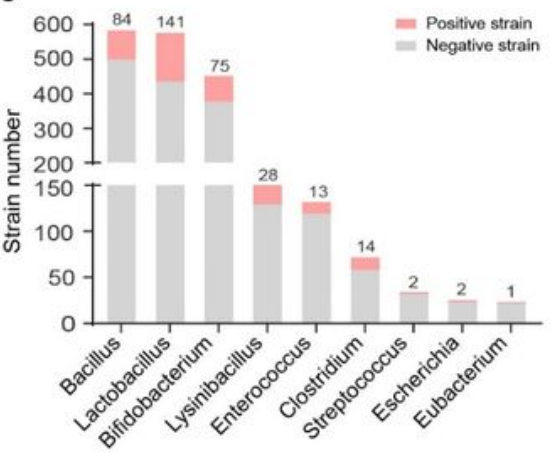

e

- Screened numbers

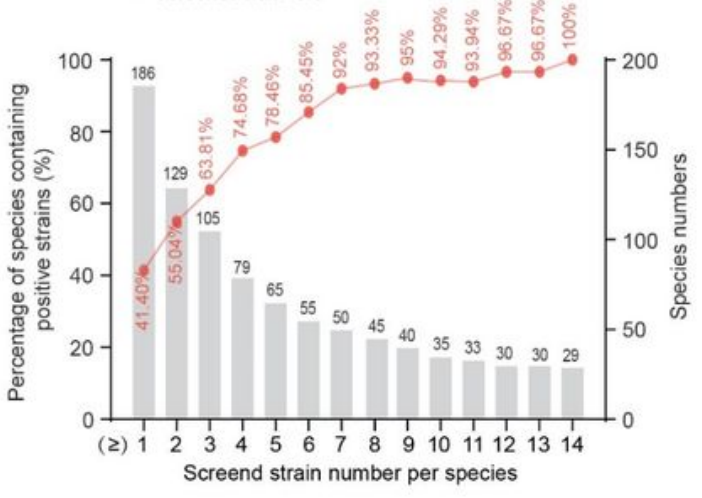

b

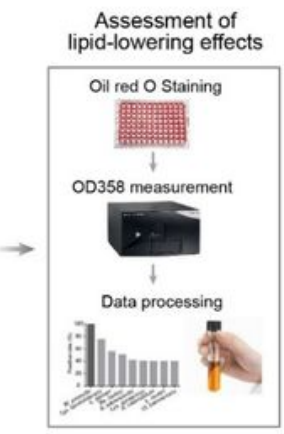

Actinobacteria Bacteroidetes

$(16.59 \%) \quad(13.16 \%)$

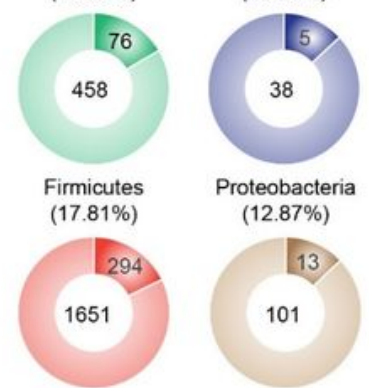

d

Genus

- Bacillus Lysinibacillus

= Bifidobacteriu

- Enterococcus = Escherichia

- Lactobacillus

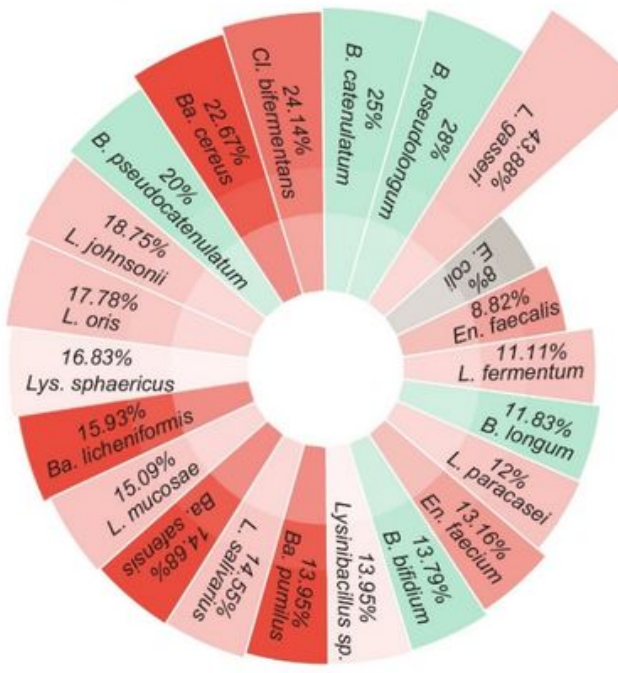

\section{Figure 1}

Strain-level screening of gut microbes for lipid-lowering activity in HepG2 cells. a Flowchart of cell-based screening for gut bacterial strains with lipid-lowering activities. $b$ and $c$ The total screened and positive strains numbers at phylum and genus levels. $d$ The rank of the positive rate for species containing $\geq 25$ tested strains in this study. e The potency of species to providing positive strains rises. The black value on the top of each bar indicates the number of species with equal or more number of tested strains in this 
study than that shown on the $x$-axis. The percentage in red indicates the rate of species providing one or more positive strains.

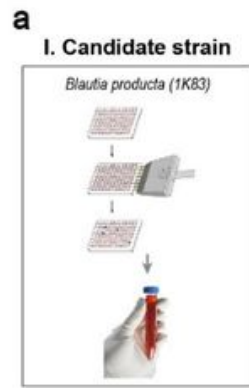

b
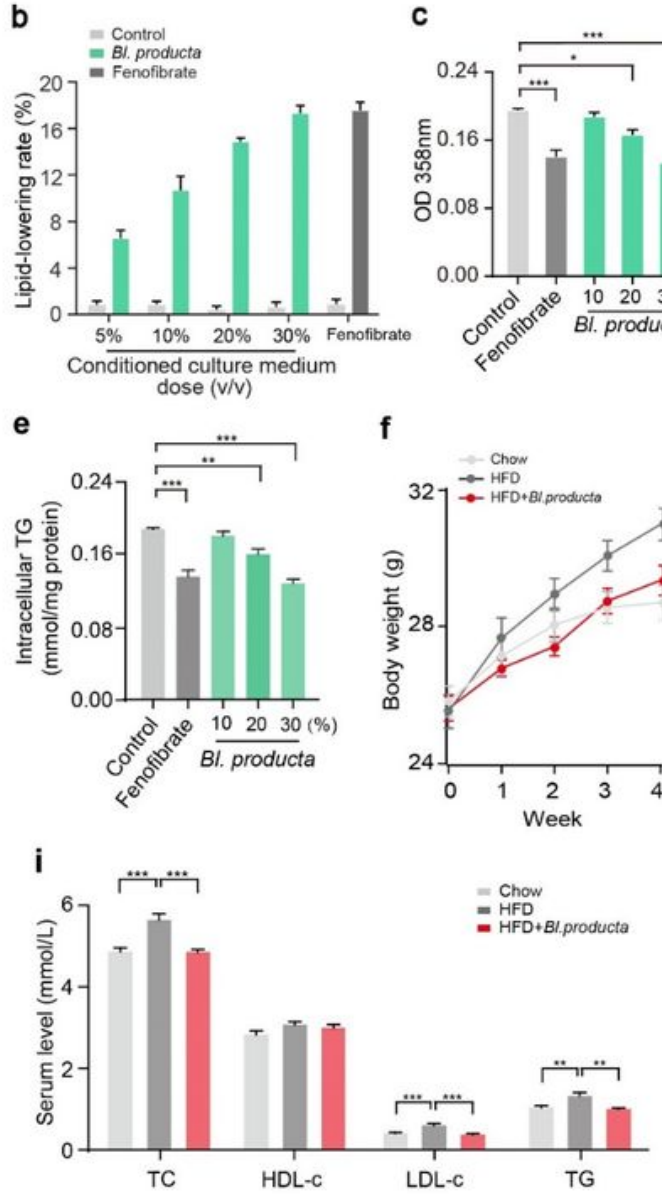

c

f
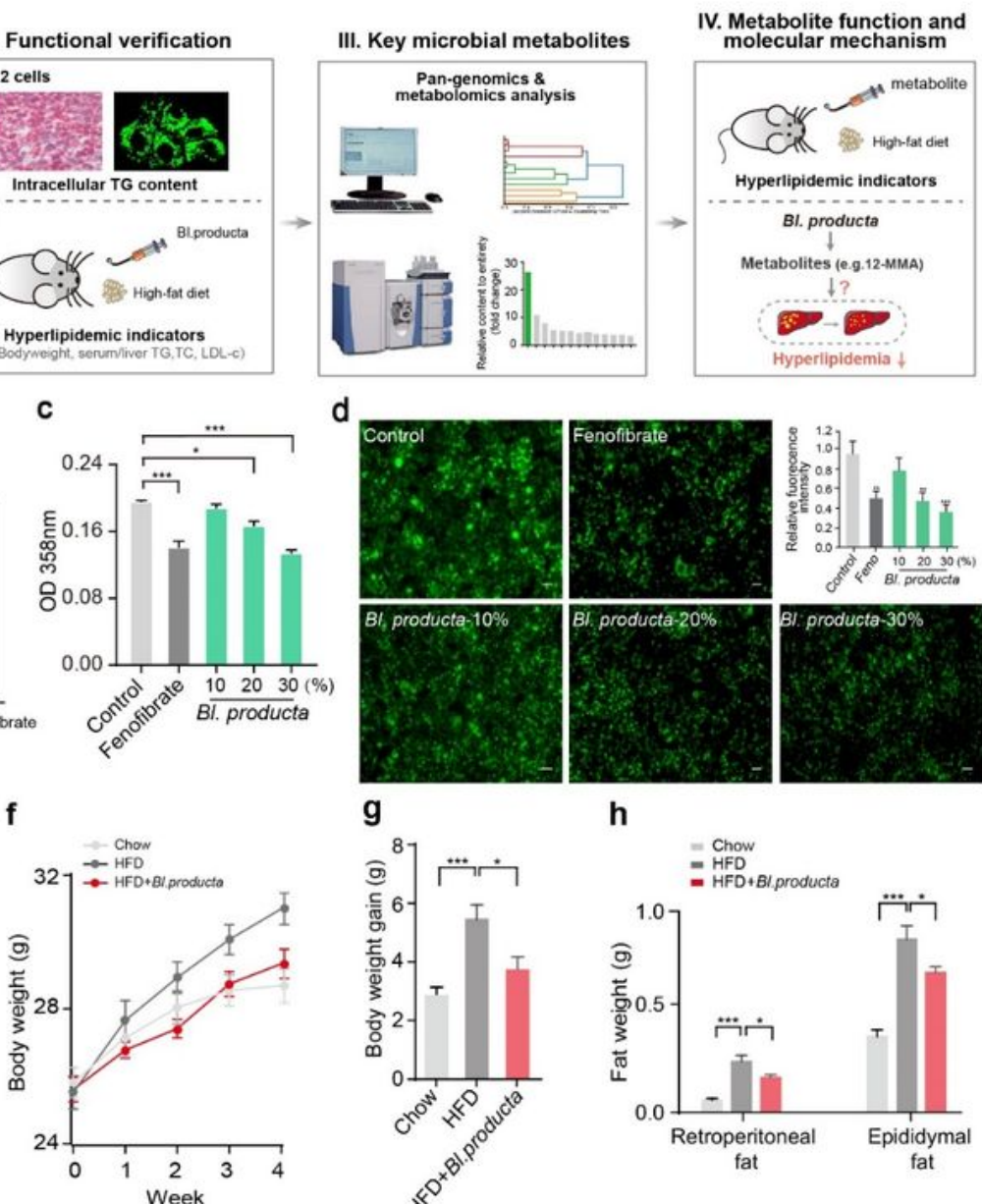

g
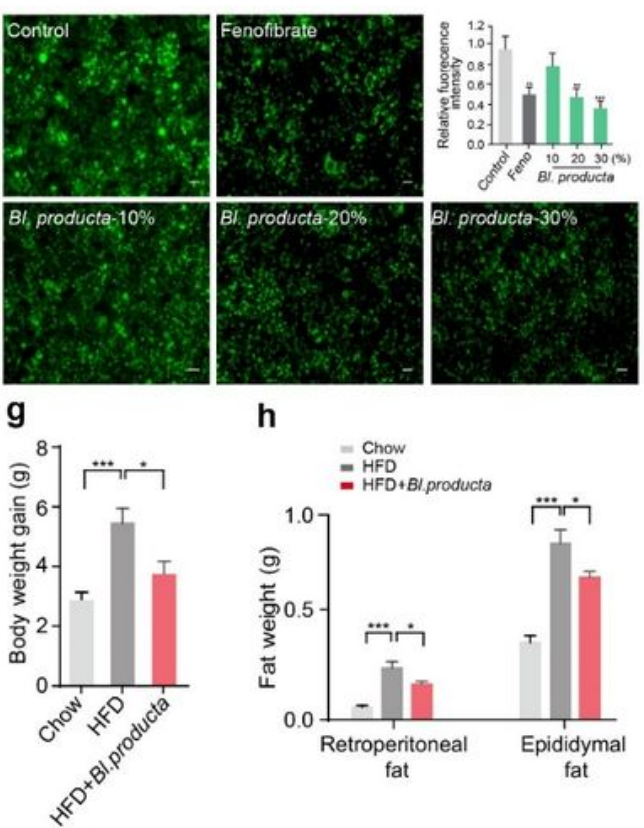

h
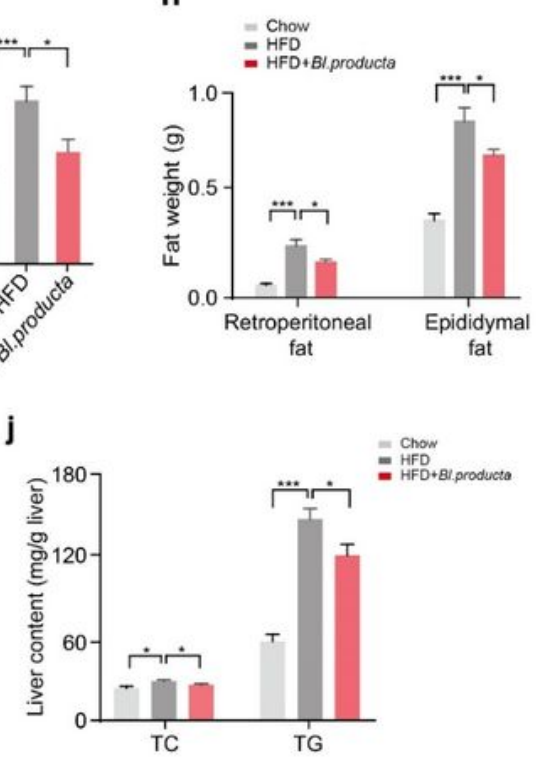

HFD
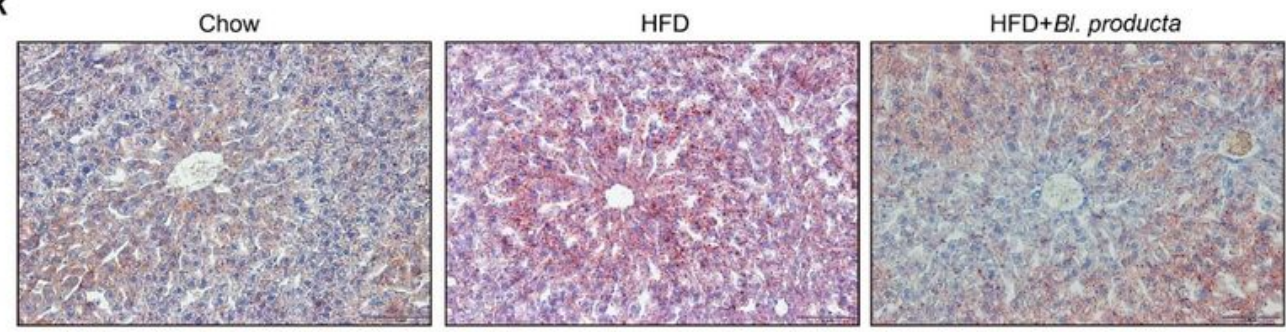

\section{Figure 2}

Blautia producta ameliorates HFD-induce hyperlipidemia. a Graphical outline for the lipid-lowering effect assessment and identification of the potential active metabolite of Bl. producta. Bl. producta strain with the best efficacy in the cell-based screening was re-assessed for the lipid-lowering effect in HepG2 cells 
and high-fat diet-induced hyperlipidemic mice. Pan-genomics and comparative metabolomics analysis were performed to find the potential active metabolites of Bl. producta. Then the anti-hyperlipidemic effect and the potential mechanism of the candidate metabolite were investigated in mice. For functional verification, the in vitro lipid-lowering effect of Bl. producta conditioned culture medium was evaluated by oil red $\mathrm{O}$ ( $\mathrm{b}$ and $\mathrm{c}$ ) and Bodipy staining, scale bars, $200 \mu \mathrm{m}$ (d), and intracellular TG quantification (e), with Fenobirate $(10 \mu \mathrm{M})$ as a positive control. The in vivo anti-hyperlipidemic effect of live Bl. producta was assessed by time-dependent bodyweight change (f), body weight gain $(\mathrm{g})$, fat weight $(\mathrm{h})$, serum lipid levels (i), liver lipid levels ( $\mathrm{j}$ ), and oil red 0 staining of liver tissue, scale bars, $100 \mu \mathrm{m}(\mathrm{k}) . \mathrm{n}=8$ for each group. ${ }^{*} P<0.05, \star \star P<<0.01,{ }^{\star \star *} P<0.001$. 

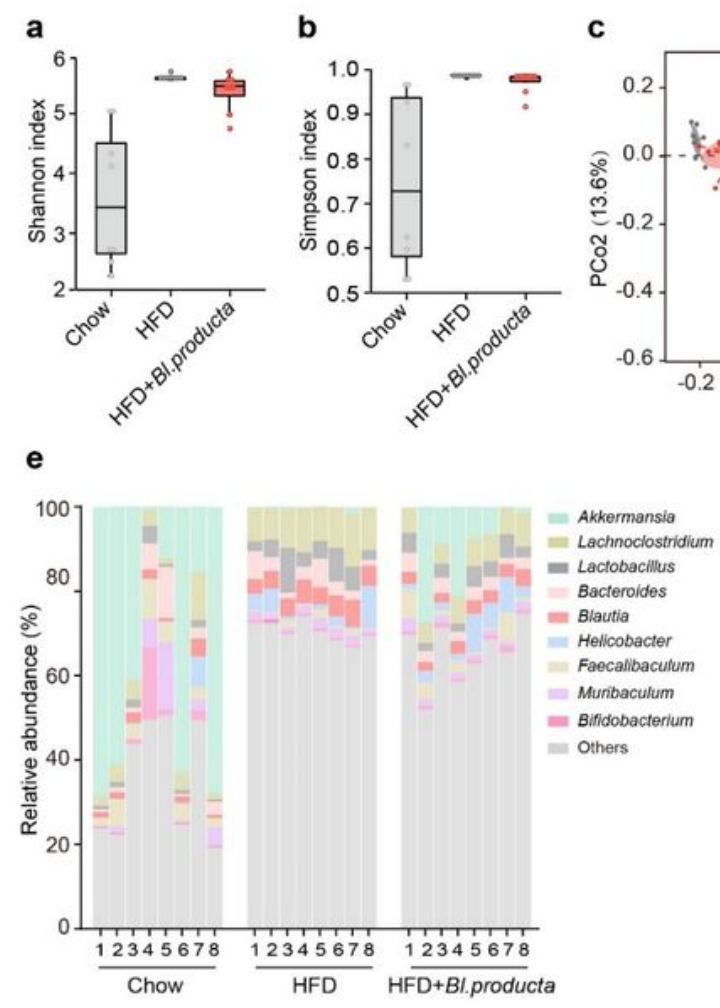

g HFD+Bl.producta

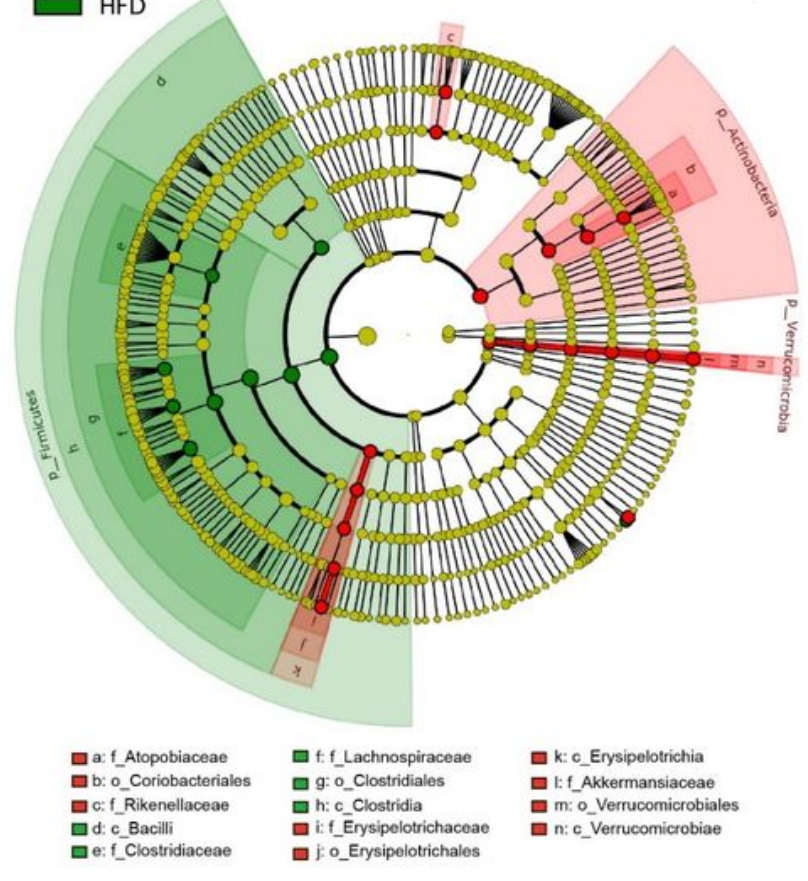

c
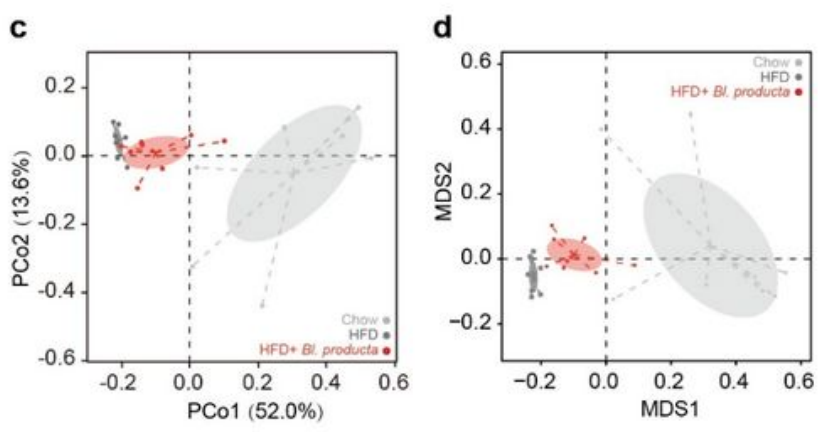

f $=$ Chow $\quad=$ HFD
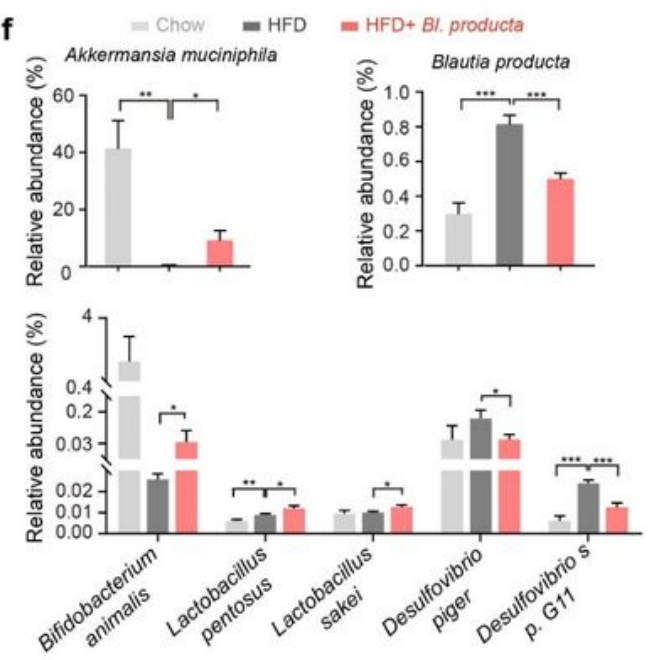

h

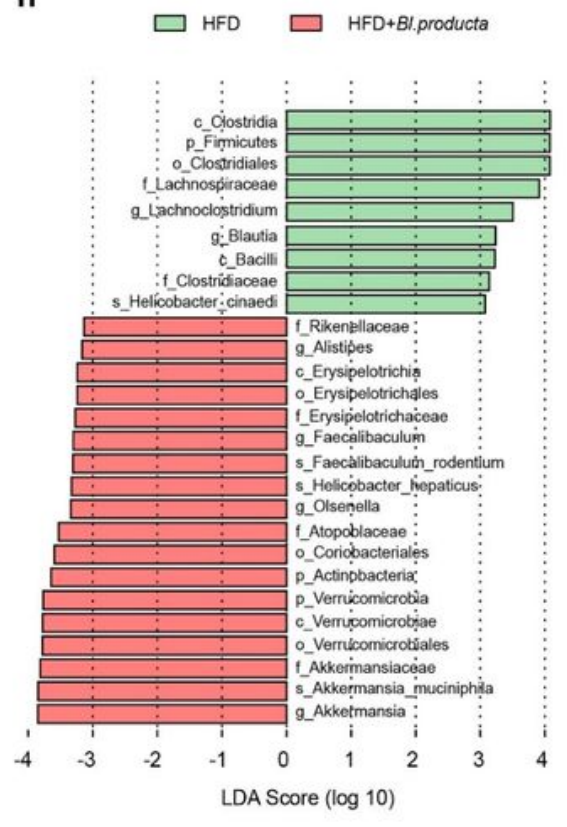

\section{Figure 3}

Blautia producta modulates gut microbiota. The alpha diversity of gut microbiota was assessed by Shannon (a) and Simpson (b) indices. The separation of the gut microbial community of different groups was assessed by principal coordinates analysis (PCoA) (c) and non-metric multi-dimensional scaling (NMDS) (d). e The profile of dominant genera in each animal. $f$ The relative abundances of BI. producta 
and significantly altered species by BI. producta. $\mathrm{g}$ Cladogram of gut microbiota community. $\mathrm{n}=8$ for each group. ${ }^{*} \mathrm{P}<0.05,{ }^{* *} \mathrm{P}<0.01,{ }^{* \star *} \mathrm{P}<0.001$.

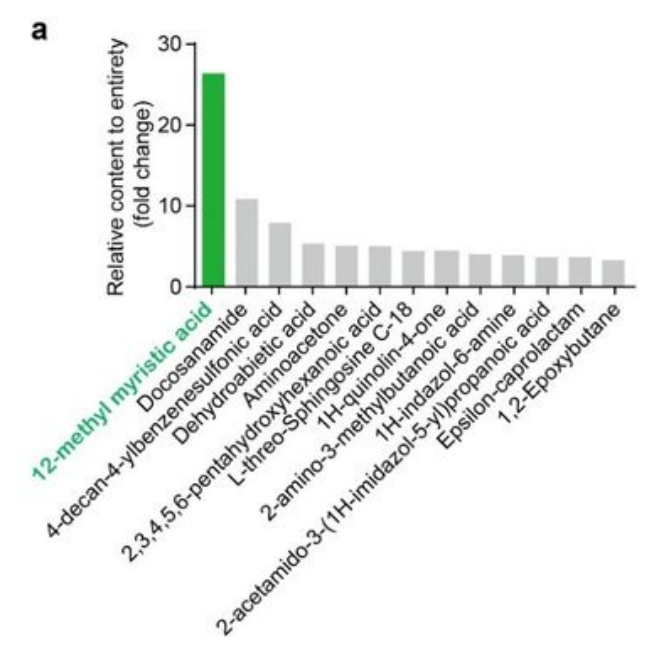

c

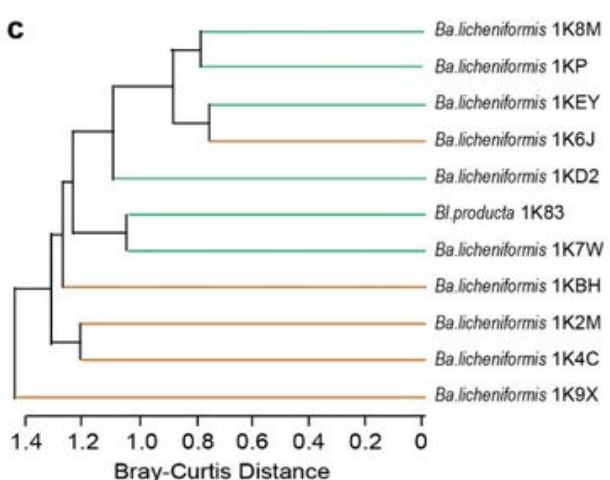

e

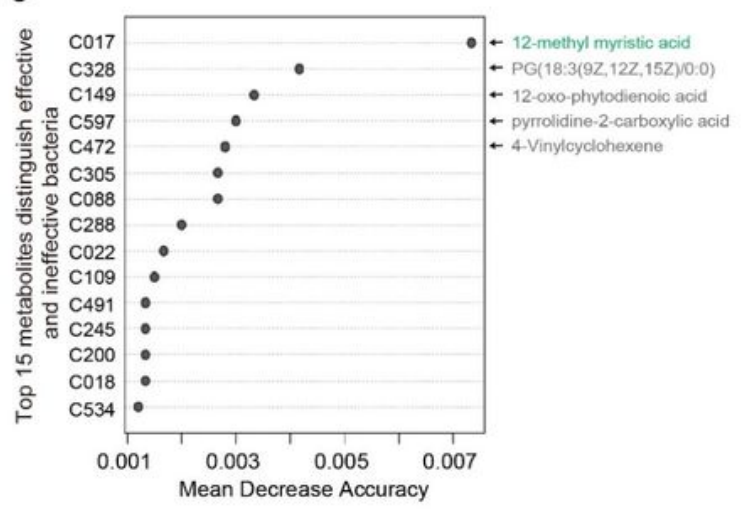

b

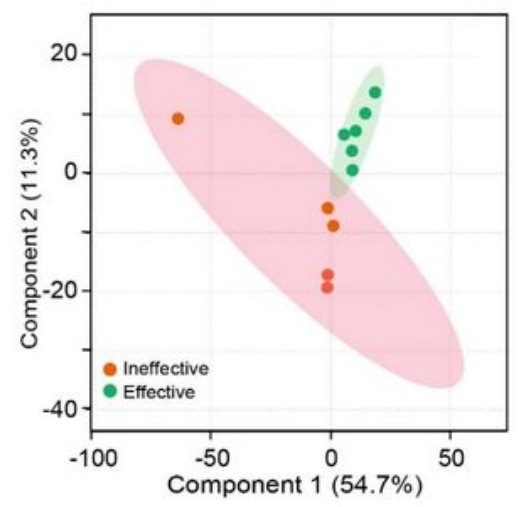

d
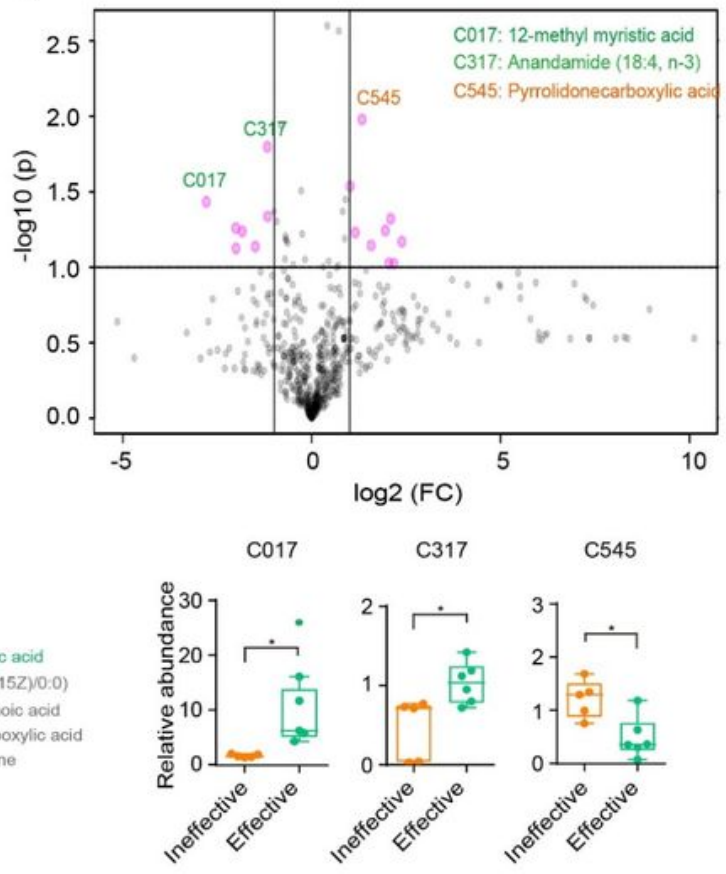

\section{Figure 4}

Metabolomic analysis identifies 12-methylmyristic acid (12-MMA) as a key active metabolite of BI. producta. a The top 13 metabolites that are markedly enriched in BI. producta. b PLS-DA plot. c Clustering analysis based on Euclidean distance. $d$ Volcano plot and the abundance of key metabolites in 
effective/ineffective stains. e The top 15 metabolites that contribute to the discrimination of effective/ineffective stains. ${ }^{*} \mathrm{P}<0.05$.

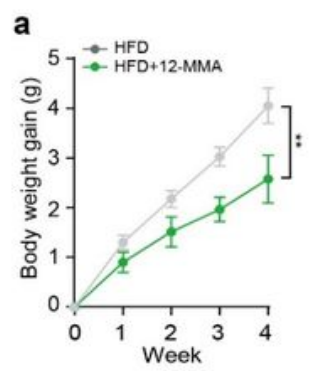

e

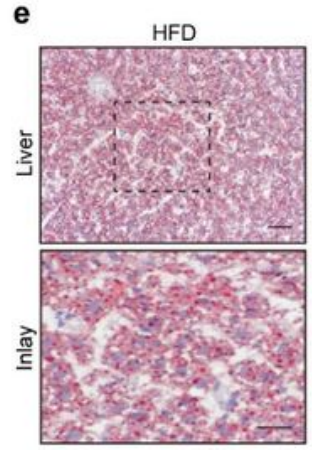

g

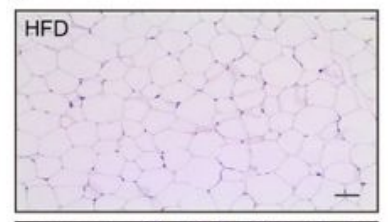

HFD + 12-MMA

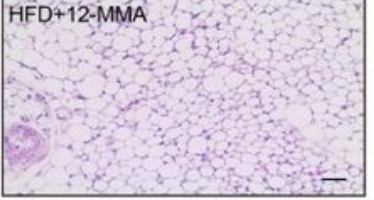

j

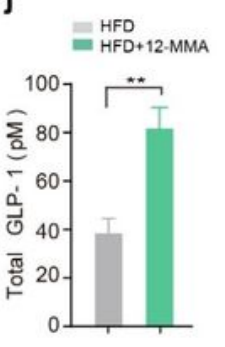

b

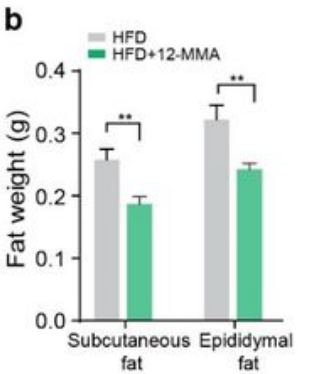

c

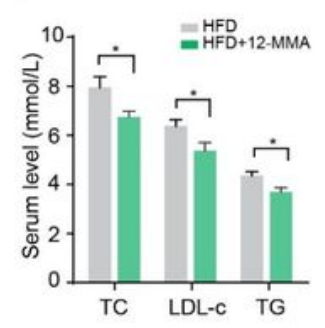

d

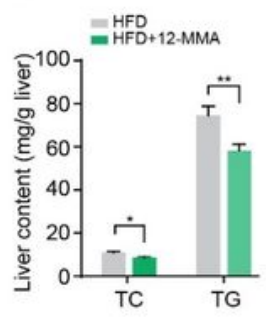

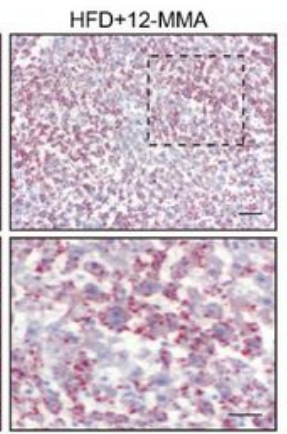

h f
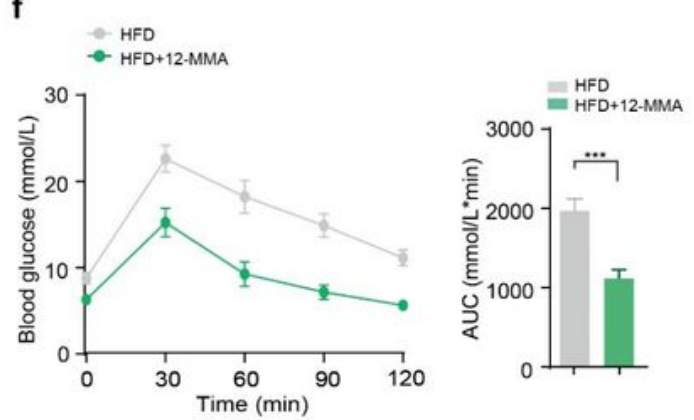

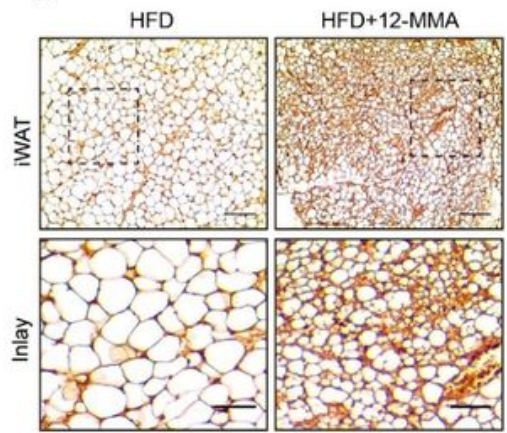

i
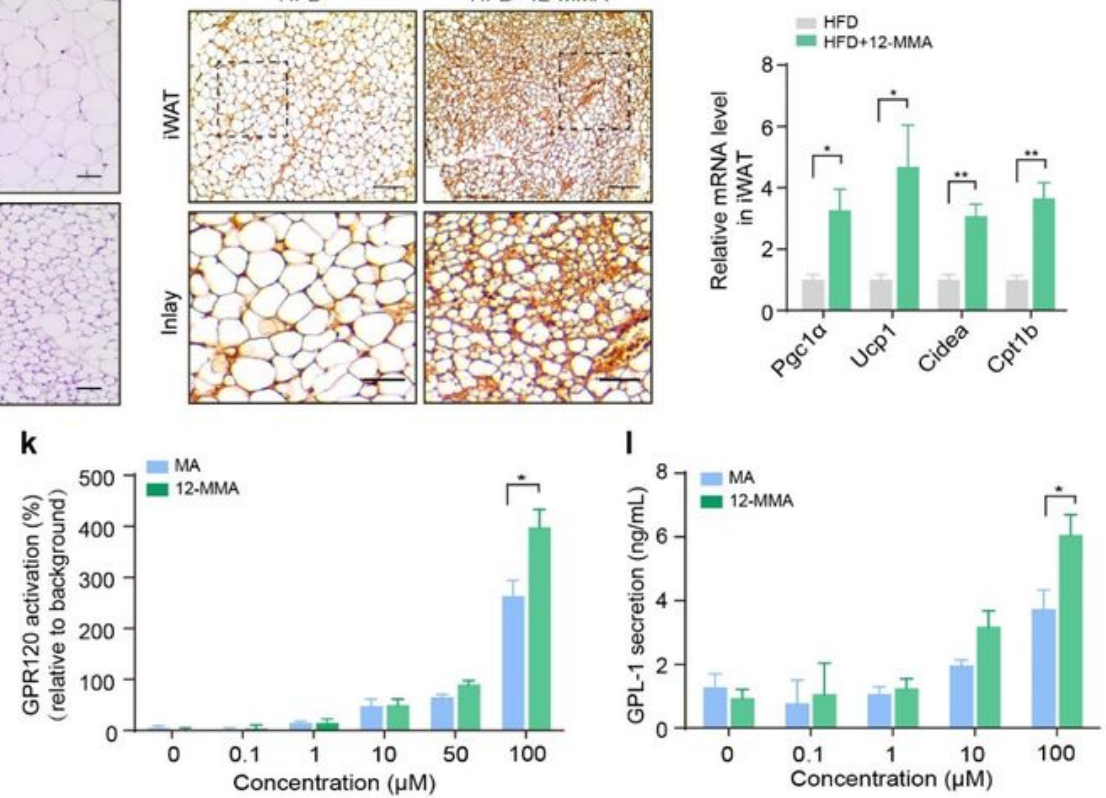

Figure 5

12-MMA effectively alleviates HFD-induced hyperlipidemia and activates GPR120. a Body weight curve of HFD-mice treated with 12-MMA or vehicle control. b Fat weight. c Serum lipids levels. d Liver lipids contents. e Oil red $\mathrm{O}$ staining of liver tissue, scale bars, $100 \mu \mathrm{m}, 50 \mu \mathrm{m}$ for inlay. F Oral glucose tolerance 
test (OGTT). g H\&E staining in inguinal white adipose tissue (iWAT), scale bars, $200 \mu \mathrm{m}$. h Ucp1 immunostaining in iWAT, scale bars, $500 \mu \mathrm{m}, 200 \mu \mathrm{m}$ for inlay. i mRNA levels of fat browning-related maker genes in iWAT. j Serum GLP-1 level. $\mathrm{n}=8$ for each animal group. k-I Fluorescence-based GPR120 activation assay and GLP-1 secretion by STC-1 intestine endocrine cells. 12-MMA was suspended in solvent ( $1 \%$ tween $80+0.5 \%$ carboxymethyl cellulose sodium (CMC-Na)) and the HFD group was treated with an equal volume of solvent. $n=6$ for each cell group. ${ }^{*} P<0.05, * * P<0.01$.

\section{Supplementary Files}

This is a list of supplementary files associated with this preprint. Click to download.

- Supplementalmaterials.pdf 